\title{
A VEHICLE PATH FOLLOWING CONTROLLER FOR COMBINED LONGITUDINAL AND LATERAL MOTION
}

\author{
Dave Mandyam ${ }^{1}$, John McPhee ${ }^{2}$, Nasser L. Azad ${ }^{3}$ \\ ${ }^{1}$ Systems Design Engineering, University of Waterloo, Waterloo, Canada \\ ${ }^{2}$ Systems Design Engineering, University of Waterloo, Waterloo, Canada \\ ${ }^{3}$ Systems Design Engineering, University of Waterloo, Waterloo, Canada
}

\begin{abstract}
Path following controllers for automobiles are frequently addressed with two separate control strategies. One of them governs longitudinal vehicle motion and the other handles supervises lateral vehicle motion. Physically, the longitudinal and lateral motions of an automobile are coupled. This coupling implies that a single controller could be designed which simultaneously handles longitudinal and lateral motion. The shared connection between longitudinal and lateral motion of a vehicle becomes critical when a vehicle is driven on slippery road conditions. Without consideration for this connection, vehicle stability controllers may fail to avoid collisions on ice. Although crucial to vehicle stability, the coupling between longitudinal and lateral motion is often ignored because its presence makes vehicle path following problems nonlinear. Given these challenges, we would like to propose a model based path following controller that combines a kinematic path following algorithm with an actuator controller for coupled vehicle motion. The kinematic path following algorithm is a vector field - based algorithm that generates a velocity vector field which directs a vehicle to a reference path defined as a contour map. If the vehicle is on the reference path, the velocity vectors become tangential to the reference path to direct the vehicle along the path. The velocity vectors are mapped to velocity states that are fed to the actuator controller to physically influence the vehicle's speed and orientation. Using a combination of feedback linearization and linear quadratic regulation, resultant forces and moments are determined to allow the vehicle's velocity to match the reference velocity states. A nonlinear least squares optimization algorithm is then applied to determine wheel torques to accelerate the vehicle based on the required resultant forces and resultant moments. This algorithm is constrained by the friction circle to ensure that maneuverability of the vehicle is maintained throughout the motion. Moreover, a kinematic steering algorithm determines steering angles at the wheels to maneuver the vehicle. Finally, the wheel torques, and steering angles are then applied to a high - fidelity vehicle model equipped with a combined slip tire force model. Path following results show the high - fidelity can smoothly merge onto paths defined as contour maps and follow those paths with small cross - track errors. We would like to consider extending this algorithm to be applicable on reference paths defined by parametric functions.
\end{abstract}

